\title{
CROPS - a decision-making aid tool for product redesign
}

\begin{abstract}
Change Ranking of Product Subsystems (CROPS) is a computer program that is designed to act as a decision-making aid for designers during early stages of their product redesign process. It generates a ranking of all subsystems within the existing product design architecture based on their estimated redesign risk. This information assists designers in selecting the right existing subsystems to be initially changed to satisfy the driving requirements, which have to be quickly decided. In this program, evaluation of redesign risk captures both direct and indirect change effects that potentially result from the modification of each subsystem. Designers are provided with a relative reference as to how risky and extensive it is to change one subsystem over the others, which can be useful when they have options to pick between several subsystems to modify for the same redesign requirement.
\end{abstract}

Keyword: Change ranking; Decision-making aid; Product redesign; Redesign risk 